\title{
Urine tenascin- $C$ is an independent risk factor for bladder cancer patients
}

\author{
ZHENFENG GUAN $^{1}$, JIN ZENG $^{2}$, ZHIQIANG WANG ${ }^{3}$, HONGJUN XIE $^{1}$, CHUAN LV $^{1}$, \\ ZHENKUN MA ${ }^{1}$, SHAN XU $^{1}$, XINYANG WANG ${ }^{1}$, DALIN HE ${ }^{2}$ and $\mathrm{LEI} \mathrm{LI}^{2}$ \\ ${ }^{1}$ Oncology Research Lab, Key Laboratory of Environment and Genes Related to Diseases, Ministry of Education; \\ ${ }^{2}$ Department of Urology, The First Affiliated Hospital of Medical College of Xi'an Jiaotong University, Xi'an, \\ Shaanxi; ${ }^{3}$ Department of Urology, The Second People's Hospital of Yinchuan City, \\ Ningxia Hui Autonomous Region, P.R. China
}

Received June 22, 2013; Accepted November 27, 2013

DOI: $10.3892 / \mathrm{mmr} .2013 .1873$

\begin{abstract}
Urine biomarkers offer a non-invasive method of detecting bladder cancer, monitoring disease progression and predicting disease recurrence and therapeutic treatment efficacy. Tenascin-C (TN-C), as a component of the extracellular matrix, is vital in the progression of bladder cancer. However, there is little to report with regard to urine $\mathrm{TN}-\mathrm{C}$ and its correlation with bladder cancer grade, stage, recurrence and prognosis. In the present study, 66 samples of voided urine from patients with bladder cancer and 42 samples from volunteers were obtained. The urine TN-C concentration was determined using an ELISA assay. The correlation between the urine $\mathrm{TN}-\mathrm{C}$ concentration and the tumor grade, stage and time from bladder cancer diagnosis to recurrence was analyzed by a rank correlation analysis. Multivariate Cox proportional hazards regression was used for finding the main life-threatening factors among age, gender, tumor grade, stage, relapse and the urine TN-C concentration. At the end, the Kaplan-Meier method was used to evaluate the survival rate affected by urine $\mathrm{TN}-\mathrm{C}$ as a single factor. The results indicated that the urine $\mathrm{TN}-\mathrm{C}$ concentration in the bladder cancer patients was higher compared with the healthy control volunteers (22.5 times higher). Among all the patients, urine TN-C concentration had a positive correlation with the bladder cancer grade and stage, with correlation coefficients of 0.905 and 0.308 , respectively; however, this correlation was negative between urine TN-C concentration and the time from bladder cancer diagnosis to recurrence. Moreover, the multivariate Cox proportional hazards model analysis indicated that urine TN-C, like tumor grade and recurrence, may be an independent risk factor for
\end{abstract}

Correspondence to: Professor Lei Li, Department of Urology, The First Affiliated Hospital of Medical College of Xi'an Jiaotong University, 277 Yanta West Road, Xi'an, Shaanxi 710061, P.R. China E-mail: dalinhexjtu@126.com

Key words: urine tenascin-C, biomarker, bladder cancer, recurrence, prognosis bladder cancer patient survival. However, it is noteworthy that inflammation may affect the concentration of urine TN-C. The results of the present study indicate that urine TN-C may be used as a biomarker for monitoring the recurrence of bladder cancer in patients and for predicting its prognosis. However, inflammation of the urinary tract should be excluded first.

\section{Introduction}

In total, $>350,000$ novel bladder cancer cases are diagnosed worldwide each year (2012), and the majority (>90\%) of these are transitional cell carcinomas (1), 70\% of which are diagnosed as superficial tumors (Ta, T1 or Tis). Despite previous refinement of various therapeutic strategies, including surgery, intravesical chemotherapy and combination therapy, 50-70\% of those superficial tumors are likely to recur (2). As bladder cancer is a relatively common and potentially life-threatening neoplasm, predicting and monitoring the condition is usually a lifelong process using complicated methods (3). Cystoscopy is the primary diagnostic method for bladder cancer as it is reasonably accurate, however, it is also invasive and relatively expensive (4). Urine cytology is highly specific in detecting bladder carcinoma, but it has a low sensitivity in detecting low- to intermediate-grade bladder cancer (5). The lifelong follow-up of patients by invasive cystoscopy with high costs and by cytology with low sensitivity emphasizes the pressing requirement for a urinary biomarker that offers a non-invasive method for the detection of recurrence and for stratifying patients with a high-risk profile.

Tenascin-C (TN-C), an extracellular matrix glycoprotein, exhibits a morphoregulatory role in fetal development and tissue remodeling (6). Recently, accumulating evidence has indicated a supportive role for TN-C in tumor growth, metastasis (7), tumor angiogenesis (8) and the inhibition of immune surveillance (6). In certain tumor types, including breast cancer, glioma and osteosarcoma, high TN-C expression provides a poor patient survival prognosis (6). In patients with bladder cancer, a diffuse TN-C staining pattern in the tumor stroma was shown to be significantly associated with a worse overall patient survival rate compared with the rate in those with moderate or negative staining, while patients with 
cytoplasmic expression of TN-C had a significantly improved overall survival rate compared with those without (9). Most significantly, only the expression of TN-C in invasive bladder cancer was an independent positive prognostic factor for the overall survival rate in the multivariate analysis (9). However, in another study using univariate and multivariate analyses, it was reported that $\mathrm{TN}-\mathrm{C}$ expression was not an independent prognostic factor for the recurrence and progression in patients with superficial bladder cancer (10). Thus, the correlation between TN-C and bladder cancer recurrence and progression remains unclear and requires further research.

Various splicing variants or splicing domains of TN-C have been demonstrated to exhibit specific biological functions during tumor progression and may have different diagnostic or predictive values (11). In breast cancer, the increased expression of TN-C splicing variants containing the B domain is positively associated with the invasive phenotype. Similarly, the A1 and $\mathrm{D}$ domains of TN-C maybe useful for determining individual bladder cancer biological behaviors (12), and detection of TN-C containing the $\mathrm{B}$ and $\mathrm{C}$ domains in urine may be used as a marker for the surveillance of bladder cancer recurrence and invasiveness $(8,9)$. However, since splicing domains may be independently included in the molecule leading to $>9$ different TN-C splicing variants, which may play different roles in cell behavior, it remains difficult to analyze all the splicing variants or splicing domains of $\mathrm{TN}-\mathrm{C}$ for diagnostic or predictive values. Additionally, one study (13) also demonstrated that it may result in wrong prognostic impressions if only one or certain domains are investigated due to the comprehensive effect of all the TN-C isoforms, which may be crucial in tumor development.

In the present study, based on the constant domains of $\mathrm{TN}-\mathrm{C}$, the concentration of urine TN-C of bladder cancer patients and volunteers was determined by ELISA analysis. We aimed to demonstrate the discrepancy of urine TN-C in bladder cancer patients vs. volunteers, and the clinical significance of this discrepancy, including tumor grade, stage and prognosis.

\section{Materials and methods}

Urine samples. In total, 66 samples of voided urine from patients with bladder cancer, 42 samples from healthy control volunteers and 33 sample from patients who suffered from urinary tract inflammation without tumors who were enrolled between Nov 2008 and Feb 2012 in the Department of Urology, First Affiliated Hospital of Medical School, Xi'an Jiaotong University (Xi'an, China) were collected. The patient's ages ranged between 32 and 82 years old, with a mean \pm standard deviation (SD) of $64.7 \pm 12.4$ years old and a male/female ratio of 44/22. The healthy volunteers had ages ranging between 35 and 75 years old, with a mean \pm SD of $65.3 \pm 7.4$ years old and a male/female ratio of 29/13. All the bladder cancer urine samples were collected following diagnosis and prior to surgery. Ethical approval for this study was obtained from the Ethics Committee of the First Affiliated Hospital of Medical School, Xi'an Jiaotong University and written informed consent was obtained from all the patients/patient's families.

ELISA analysis. The ELISA kits were obtained from Shanghai Westang Biological Technology Co., Ltd. (Shanghai, China)
Table I. Epidemiology and clinicopathology of bladder cancer patients and volunteers.

\begin{tabular}{lccc}
\hline Data & Patients & Volunteers & $\begin{array}{c}\text { Inflammation } \\
\text { patients }\end{array}$ \\
\hline Gender, n (\%) & & & \\
Male & $44(66.7)$ & $29(69.0)$ & $18(56)$ \\
Female & $22(33.3)$ & $13(31.0)$ & $15(44)$ \\
Age, years & & & \\
Range & $32-82$ & $35-75$ & $29-61$ \\
Mean \pm SD & $64.7 \pm 12.4$ & $65.3 \pm 7.4$ & $34.7 \pm 5.5$ \\
Tumor grade, n (\%) & & & - \\
I & $22(33.3)$ & - & - \\
II & $25(37.9)$ & - & - \\
III & $19(28.8)$ & - & - \\
Tumor stage, n $(\%)$ & & - & - \\
Ta, Tis & $11(16.7)$ & - & - \\
T1 & $23(34.8)$ & - & - \\
T2 & $23(34.8)$ & - & - \\
T3 & $9(13.6)$ & - & \\
\hline
\end{tabular}

Epidemiology and clinicopathology of bladder cancer patients and volunteers. In total, 66 bladder cancer patients and 42 volunteers were enrolled. In the present study, volunteers included individuals with no bladder cancer or inflammatory diseases. Inflammation patients were the patients who suffered from urinary tract inflammation without tumor diseases. Tumor grade and tumor stage classification was according to the standard Tumor Node Metastasis WHO criteria of 1973 and 2002, respectively (14). SD, standard deviation.

to target the constant domain of TN-C. The lowest concentration that may be detected by the kit is $<2 \mathrm{ng} / \mathrm{ml}$. ELISA analysis was performed by a blinded investigator and each sample was analyzed at least in triplicate. Among all 66 patients, 48 were followed up for their life status until the end of the study.

Statistical analysis. Student's t-test was used for analyzing the differences in the TN-C concentrations between the patients and volunteers. A rank correlation was used for evaluating the correlation between the urine $\mathrm{TN}-\mathrm{C}$ concentration and the tumor grade/stage and the time from bladder cancer diagnosis to recurrence. A multivariate Cox proportional hazards model was used to analyze the main hazard factor of survival rate among other factors of age, relapse, gender, grade, stage and concentration of TN-C, and the Kaplan-Meier method was used for the survival rate analysis. A value of $\mathrm{P}<0.05$ was considered to indicate a statistically significant difference.

\section{Results}

Urine concentration of TN-C in bladder cancer patients is higher than in volunteers. All the 66 bladder cancer patients had been diagnosed with bladder cancer for the first time and they had no history of cancerous disease. The clinicopathological data of the bladder cancer patients is listed in 
Table II. Urine concentration of TN-C in bladder cancer patients and volunteers $(\mu \mathrm{g} / \mathrm{ml})$.

\begin{tabular}{lccccc}
\hline Patients & Total number & Gender, $\mathrm{n}(\mathrm{M} / \mathrm{F})$ & Age range, years & Age, years (mean $\pm \mathrm{SD})$ & $\mathrm{TN}-\mathrm{C}, \mu \mathrm{g} / \mathrm{ml}(\mathrm{mean} \pm \mathrm{SD})$ \\
\hline Bladder cancer & 66 & $44 / 22$ & $32-82$ & $64.7 \pm 12.4$ & $0.0630 \pm 0.0331^{\mathrm{a}}$ \\
Volunteers & 42 & $29 / 13$ & $35-75$ & $65.3 \pm 7.4$ & $0.0028 \pm 0.0026$
\end{tabular}

${ }^{\mathrm{a}} \mathrm{P}<0.001$. Urine concentration of TN-C in bladder cancer patients and volunteers $(\mu \mathrm{g} / \mathrm{ml})$. As shown, the urine TN-C concentration of the bladder cancer patients was significantly higher compared with the volunteers. TN-C, tenascin-C; M, male; F, female; SD, standard deviation.

Table III. Urine TN-C in different grades and stages of bladder cancer.

\begin{tabular}{lcccc}
\hline Tumor characteristics & $\mathrm{n}$ & Age, years (mean $\pm \mathrm{SD})$ & Gender, $\mathrm{n}(\mathrm{M} / \mathrm{F})$ & $\mathrm{TN}-\mathrm{C}, \mu \mathrm{g} / \mathrm{ml}(\mathrm{mean} \pm \mathrm{SD})$ \\
\hline Stage & & & $17 / 6$ & $0.0593 \pm 0.0379$ \\
T1 & 23 & $63.7 \pm 11.7$ & $14 / 9$ & $0.0659 \pm 0.0259^{\mathrm{a}}$ \\
T2 & 23 & $65.4 \pm 12.2$ & $6 / 3$ & $0.0916 \pm 0.0263^{\mathrm{b}}$ \\
T3 & 9 & $71.2 \pm 6.1$ & $8 / 3$ & $0.0412 \pm 0.0251$ \\
Ta, Tis & 11 & $60.1 \pm 16.5$ & & $0.0299 \pm 0.0100$ \\
Grade & & & $14 / 8$ & $0.0592 \pm 0.0155^{\mathrm{c}}$ \\
1 & 22 & $65.0 \pm 12.0$ & $17 / 8$ & $0.1061 \pm 0.0141^{\mathrm{d}}$ \\
2 & 25 & $64.4 \pm 14.1$ & $14 / 5$ & 0 \\
3 & 19 & $64.8 \pm 10.9$ & & 0 \\
\hline
\end{tabular}

Correlation analysis of urine $\mathrm{TN}-\mathrm{C}$ concentration with bladder cancer grade/stage and the time from diagnosis to recurrence. The differences in urine $\mathrm{TN}-\mathrm{C}$ concentration in different bladder cancer grade and stage are significant $(\mathrm{P}<0.05)$. ${ }^{\mathrm{a}} \mathrm{P}=0.011,{ }^{\mathrm{b}} \mathrm{P}=0.009,{ }^{\mathrm{c}} \mathrm{P}=0.001$ and ${ }^{\mathrm{d}} \mathrm{P}=0.0000392$. The discrepancy of urine TN-C in different bladder cancer grade and stage are significant, $\mathrm{P}<0.05$. TN-C, tenascin-C; $\mathrm{SD}$, standard deviation .

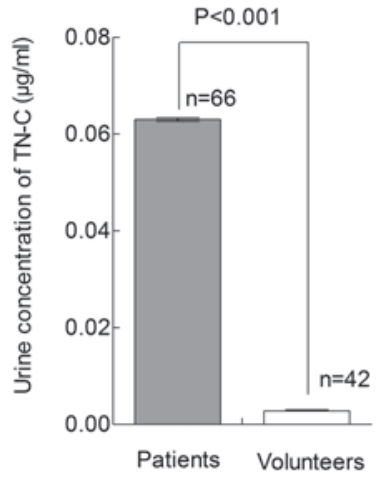

Figure 1. Urine concentration of TN-C in bladder cancer patients and volunteers. Urine $\mathrm{TN}-\mathrm{C}$ in bladder cancer patients was observed to be markedly higher compared with the volunteers, $\mathrm{P}<0.000$. TN-C, tenascin-C.

Table I. The mean concentration of the $\mathrm{TN}-\mathrm{C}$ in the voided urine was $0.0630 \pm 0.0331$ vs. $0.0028 \pm 0.0026$ for the bladder cancer patients vs. the healthy control volunteers, respectively $(\mathrm{P}<0.001)$. The t-test analysis indicated that between the two groups, the concentration of TN-C in their voided urine was markedly different, as shown in Table II and Fig. 1. The results of the present study indicated that the bladder cancer patients had a higher concentration of urine $\mathrm{TN}-\mathrm{C}$ compared with the healthy volunteers. However, as reported, our results also provide evidence that inflammation contributes to the elevation of urine TN-C (Table VI).
Urine concentration of $T N-C$ has a positive correlation with bladder cancer grade and clinical stage. In order to illustrate the correlation between urine $\mathrm{TN}-\mathrm{C}$ and tumor grade/tumor stage, the urine TN-C concentrations for differing grades and stages are listed, as shown in Table III. From grades 1-3, the urine TN-C concentration (mean $\pm \mathrm{SD}$ ) was $0.0299 \pm 0.0100$, $0.0592 \pm 0.0155$ and $0.1061 \pm 0.0141 \mu \mathrm{g} / \mathrm{ml}$, respectively $(\mathrm{P}<0.05)$, however, from stages $\mathrm{T} 1$ to $\mathrm{T} 3$, the urine $\mathrm{TN}-\mathrm{C}$ concentration was $0.0593 \pm 0.0379,0.0659 \pm 0.0259$ and $0.0916 \pm 0.0263 \mu \mathrm{g} / \mathrm{ml}$, respectively $(\mathrm{P}<0.05)$. The rank correlation analysis indicated that the coefficient between the concentration of TN-C and bladder cancer grade was 0.9050 , and this value in bladder cancer stage was 0.3080 , which indicates that the urine concentration of $\mathrm{TN}-\mathrm{C}$ had a more significantly positive correlation with the tumor grade compared with the tumor stage; the P-value was 0.0000103 and 0.0220034 , respectively, as shown in Table IV. These results indicate that the urine concentration of TN-C has a positive correlation with the tumor grade and tumor stage, particularly the former.

Urine concentration of $T N-C$ has a negative correlation with the time from bladder cancer diagnosis to recurrence. In order to illustrate the correlation between the urine concentration of TN-C and the time from bladder cancer diagnosis to recurrence, 48 bladder cancer patients were followed up. In total, 16 of these patients exhibited cancer recurrence (33\%), and during the follow-up stage, all 16 patients only had one recurrence. The rank correlation analysis indicated that the 
Table IV. Correlation analysis of urine TN-C concentration with bladder cancer grade/stage and the time from diagnosis to recurrence.

\begin{tabular}{lcc}
\hline Tumor characteristics & Correlation coefficient with urine TN-C & P-value \\
\hline Bladder cancer grade & 0.905 & 0.0000103 \\
Bladder cancer stage & 0.308 & 0.0220034 \\
Time from diagnosis to recurrence & -0.874 & 0.0000796
\end{tabular}

Correlation coefficient of urine TN-C and bladder cancer grade/stage is $0.905 / 0.308$, respectively, while this coefficient is -0.874 in the time from bladder cancer diagnosis to recurrence. In the tumor stage, the total number of doses was not included in the Ta and Tis stage during the correlation analysis. TN-C, tenascin-C.

Table V. Results of the Cox proportional hazards analysis for the main risk factor for bladder cancer patients [-2ln $(\mathrm{L})=126.86]$.

\begin{tabular}{|c|c|c|c|c|c|c|c|c|}
\hline \multirow[b]{2}{*}{ Variable } & \multirow[b]{2}{*}{$\mathrm{df}$} & \multirow[b]{2}{*}{$\mathrm{b}$} & \multirow[b]{2}{*}{$\mathrm{SE}(\mathrm{b})$} & \multirow[b]{2}{*}{ Wald $\chi^{2}$} & \multirow[b]{2}{*}{ P-value } & \multirow[b]{2}{*}{ RR } & \multicolumn{2}{|c|}{$95 \% \mathrm{CI}$ for RR } \\
\hline & & & & & & & Upper & Lower \\
\hline Relapse & 1 & -1.630 & 0.536 & 9.243 & 0.002 & 0.196 & 0.068 & 0.560 \\
\hline TN-C & 1 & 0.658 & 0.198 & 11.068 & 0.001 & 1.931 & 1.311 & 2.846 \\
\hline Grade & 1 & 0.570 & 0.281 & 4.114 & 0.043 & 1.768 & 1.019 & 3.067 \\
\hline
\end{tabular}

The cox proportional hazards analysis to find the main risk factors for bladder cancer patients. Multivariate Cox stepwise regression was performed. The relative ratio (RR) values were $0.196,1.931$ and 1.768 for relapse, urine TN-C concentration and tumor grade, respectively. TN-C, tenascin-C; $\mathrm{CI}$, confidence interval; degrees of freedom; b, estimates of the regression coefficient; $\mathrm{SE}(\mathrm{b})$, standard error of regression coefficient of estimates

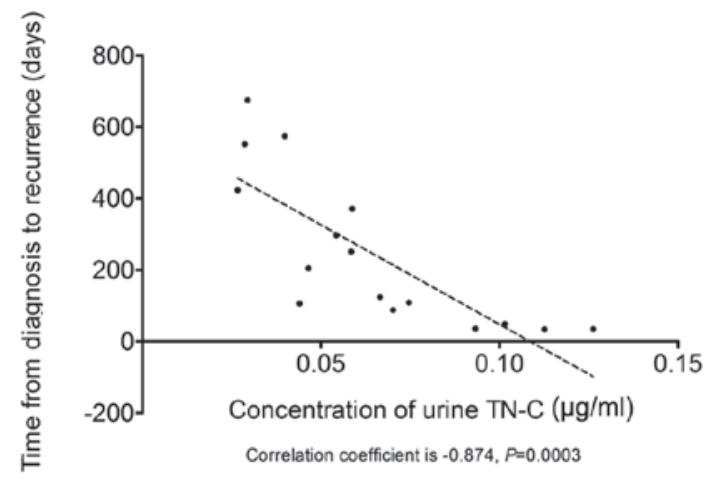

Figure 2. Correlation analysis for the urine TN-C and the time from bladder cancer diagnosis to recurrence. The $\mathrm{X}$-axis indicates the concentration of urine $\mathrm{TN}-\mathrm{C}$, the $\mathrm{y}$-axis indicates the time from bladder cancer diagnosis to its first recurrence. With the increase of urine $\mathrm{TN}-\mathrm{C}$, the time from diagnosis to recurrence is decreased, and the coefficient of this negative correlation is $-0.874, \mathrm{P}=0.0003$. TN-C, tenascin-C.

coefficient between the urine concentration of TN-C and the time from bladder cancer diagnosis to recurrence was -0.8740 $(\mathrm{P}=0.0003)$, as shown in Table IV and Fig. 2, indicating that urine $\mathrm{TN}-\mathrm{C}$ concentration has a negative correlation with the time from bladder cancer diagnosis to recurrence.

Urine TN-C is one of the hazard factors for the prognosis of bladder cancer patients. Among the 48 bladder cancer patients who were followed up, 24 succumbed to bladder cancer (50\%), 16 suffered recurrence of the disease $(33 \%)$ and the other 8 patients remained alive without bladder cancer (16.7\%). In the present study, factors, including tumor grade, stage, age and

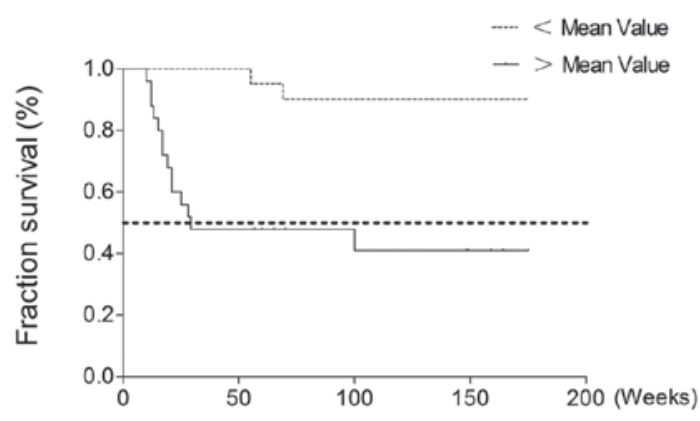

Figure 3. Survival rate caused by urine TN-C as an independent factor. The 48 patients were divided into two groups according to their mean urine TN-C concentration, and the survival rate was indicated by Kaplan-Meier analysis. The survival rate of the two groups are markedly different, indicating that urine TN-C is an independent factor for bladder cancer patients. TN-C, tenascin-C.

gender of the patient, recurrence and the urine concentration of TN-C, were analyzed to evaluate the risk factors for the survival rate of the patients with bladder cancer. The urine concentration of TN-C was divided into four grades denoted $1,2,3$ and 4 according to their percentile: 1 , min-X25\%; 2, $\mathrm{X} 25-\mathrm{X} 50 \%$; 3, X50-X75\%; and 4, 75\%-max (X represents the concentration of urine $\mathrm{TN}-\mathrm{C}$ ). The Cox proportional hazards analysis revealed that among the six factors, bladder cancer grade, recurrence and urine $\mathrm{TN}-\mathrm{C}$ were the independent risk factors for the bladder cancer patients, as shown in Table V; the relative ratio (RR) values were $1.7680,0.1960$ and 1.9310 , respectively. The survival rate, as affected by urine TN-C concentration, was then focused on as a single risk factor in 
Table VI. Urine TN-C in patients with inflammation compared with healthy volunteers and bladder cancer patients.

Patient groups

Bladder cancer patients

Inflammation patients

Healthy volunteers
Total sample number

$\mathrm{TN}-\mathrm{C}, \mu \mathrm{g} / \mathrm{ml}($ mean $\pm \mathrm{SD})$

${ }^{a} \mathrm{P}=0.0009$. In order to validate whether inflammation has effects on the urine TN-C, 33 urinary tract inflammation patients were enrolled into the study, and these 33 patients were diagnosed with urinary tract inflammation without any other disease. The urine samples were collected and analyzed in the same manner as the urine samples from the bladder cancer patients and volunteers and by the same investigator. As shown, the result indicates that the urine TN-C concentration in the inflammation patients was significantly elevated $(\mathrm{P}=0.0009)$. TN-C, tenascin- $\mathrm{C}$; $\mathrm{SD}$, standard deviation.

the 48 bladder cancer patients. The patients were divided into two groups according to whether their urine TN-C concentration was above or below the mean study value $(0.0690 \mu \mathrm{g} / \mathrm{ml})$, as shown in Fig. 3. The survival rate between the two groups was observed to be significantly different, indicating that urine $\mathrm{TN}-\mathrm{C}$ is an independent risk factor for bladder cancer patients.

\section{Discussion}

The most difficult obstacle to the therapeutics of bladder cancer is the high rate of tumor recurrence and the absence of specific manifestations to monitor using cystoscopy following surgery. Due to the limitations of invasive and expensive cystoscopy (15), numerous urine marker-based tests for bladder cancer have been developed and tested in different populations in the last decades (16), including cytology, ImmunoCyt ${ }^{\mathrm{TM}}$, fluorescence in situ hybridization, proteomics, microsatellite analysis and biomarkers, including BTAstat, BTAtrak, NMP22 (17), FDP, Engrailed-2 (18), Apo-I (19) and UPK3A (15). However, all these biomarkers have their own limitations and must be combined with other biomarkers in order to monitor bladder cancer (20). Currently, no single urine marker guides us in monitoring bladder cancer perfectly, and a set of novel markers are required.

$\mathrm{TN}-\mathrm{C}$ is a main component of the extracellular matrix in solid tumor tissues (21), and is a molecule of $\sim 300 \mathrm{kDa}$ as an intact monomer and up to $1,800 \mathrm{kDa}$ when assembled into a hexamer. The multi-domain molecule consists of an N-terminal assembly domain, followed by 14.5 EGF-like repeats, 8 constant and $\leq 9$ alternatively spliced fibronectin type III repeats and a C-terminal fibrinogen-like globular domain (6); this special mRNA and protein structure is the reason for its complicated and multiple functions, which involve adhesion/anti-adhesion, epithelial-mesenchymal transition, proliferation and cell cycle control (6). A growing body of evidence has indicated the vital role of TN-C in breast (7), pancreatic $(22)$, prostate $(23,24)$ and lung cancers $(25)$ as well as squamous cell carcinoma of the head and neck (26). In bladder cancer, TN-C has been reported to contribute to migration and invasion (9,27); this function depends on its isoforms, which are produced by divergent splicing of the alternative domains, and those isoforms may also be regarded as prognostic markers of bladder cancer $(12,28)$. In the present study, ELISA kits were used to target the constant splicing domains in order to detect all the isoforms of TN-C in the urine samples. To the best of our knowledge, this is the first study focused on all the isoforms of urine TN-C and the correlation between urine $\mathrm{TN}-\mathrm{C}$ and bladder cancer grade/stage. The urine $\mathrm{TN}-\mathrm{C}$ concentration was revealed to be positively associated with the tumor stage and histological grade, which is consistent with a previous study performed in bladder cancer tissues (28). Additionally, the rank correlation analysis also indicated that the urine $\mathrm{TN}-\mathrm{C}$ concentration had a negative correlation with the time from bladder cancer diagnosis to recurrence, indicating that a higher urine TN-C concentration may be a predictor for bladder cancer recurrence. Furthermore, the Cox proportional hazards analysis revealed that urine $\mathrm{TN}-\mathrm{C}$ may be regarded as an independent risk factor for the prognosis of bladder cancer, and the Kaplan-Meier analysis indicated that urine TN-C, as a single risk factor, is vital in the survival rate of bladder cancer patients.

The present study mainly focused on the total isoforms of TN-C containing the constant domain, but not the specific alternative domain, which is different from the studies previously reported (29). Based on numerous investigations, the superiority of the present study may be attributed to the following points: The exact numbers of functional isoforms that contribute to the progression of bladder cancer remain unclear. The functional isoforms of $\mathrm{TN}-\mathrm{C}$ may be far beyond the numbers observed in this study. Therefore, targeting one or several specific isoforms of urine TN-C may lead to an underestimation of the actual correlation between $\mathrm{TN}-\mathrm{C}$ and cancer progression. Furthermore, the concentration of urine TN-C is measured at the nanogram level. Detection of the specific isoforms of TN-C magnifies the random error on this level, since it is too low to be detected perfectly. Detection targeting to the isoform containing the constant domain may provide more accurate results due to its higher concentration compared with any specific isoforms. Additionally, the specificity of the antibodies targeting specific isoforms may also result in false-positive or false-negative errors.

There is another point that is worth noting. The expression of TN-C may be affected by numerous factors (6), including wound healing and inflammation, and the data in Table VI also indicated that the urine TN-C of the patients with infection of the urinary tract was significantly elevated. Therefore, a diagnosis of inflammation/infection must first be excluded 
when using the urine $\mathrm{TN}-\mathrm{C}$ concentration for predicting the prognosis of the bladder cancer patients.

In conclusion, the present study indicated that the voided urine concentration of $\mathrm{TN}-\mathrm{C}$ in bladder cancer patients is significantly elevated and correlated with the progression of the tumor grade and stage, and that elevated urine TN-C concentration may be an independent life-threatening factor for bladder cancer patients. The present study thus provides a potentially useful prognostic marker for patients with bladder cancer and indicates that TN-C concentration merits further investigation.

\section{Acknowledgements}

This study was partly supported by the National Natural Science Foundation of China (grant no. 81101936).

\section{References}

1. Griffiths TR; Action on Bladder Cancer: Current perspectives in bladder cancer management. Int J Clin Pract 67: 435-448, 2013.

2. Kaufman DS, Shipley WU and Feldman AS: Bladder cancer Lancet 374: 239-249, 2009.

3. Al Hussain TO and Akhtar M: Molecular basis of urinary bladder cancer. Adv Anat Pathol 20: 53-60, 2013.

4. Kausch I and Böhle A: Bladder cancer. II. Molecular aspects and diagnosis. Eur Urol 39: 498-506, 2001.

5. Lokeshwar VB, Schroeder GL, Selzer MG, et al: Bladder tumor markers for monitoring recurrence and screening comparison of hyaluronic acid-hyaluronidase and BTA-Stat tests. Cancer 95: 61-72, 2002.

6. Orend $\mathrm{G}$ and Chiquet-Ehrismann R: Tenascin-C induced signaling in cancer. Cancer Lett 244: 143-163, 2006.

7. Oskarsson T, Acharyya S, Zhang XH, et al: Breast cancer cells produce tenascin $\mathrm{C}$ as a metastatic niche component to colonize the lungs. Nat Med 17: 867-874, 2011.

8. Berndt A, Köllner R, Richter P, et al: A comparative analysis of oncofetal fibronectin and tenascin-C incorporation in tumour vessels using human recombinant SIP format antibodies. Histochem Cell Biol 133: 467-475, 2010.

9. Brunner A, Mayerl C, Tzankov A, et al: Prognostic significance of tenascin- $\mathrm{C}$ expression in superficial and invasive bladder cancer. J Clin Pathol 57: 927-931, 2004.

10. Ioachim E, Michael M, Stavropoulos NE, Kitsiou E, Salmas M and Malamou-Mitsi V: A clinicopathological study of the expression of extracellular matrix components in urothelial carcinoma. BJU Int 95: 655-659, 2005.

11. Hancox RA, Allen MD, Holliday DL, et al: Tumour-associated tenascin-C isoforms promote breast cancer cell invasion and growth by matrix metalloproteinase-dependent and independent mechanisms. Breast Cancer Res 11: R24, 2009.

12. Wunderlich H, Berndt A, Anger K, et al: A1 and D domain of tenascin $\mathrm{C}$ - New prognostic marker in bladder cancer. J Urology 179: 315, 2008.
13. FischerD,Brown-LüdiM,SchulthessTandChiquet-Ehrismann-R: Concerted action of tenascin- $\mathrm{C}$ domains in cell adhesion, antiadhesion and promotion of neurite outgrowth. J Cel Sci 110: 1513-1522.

14. Babjuk M, Oosterlinck W, Sylvester R, et al: EAU guidelines on non-muscle-invasive urothelial carcinoma of the bladder. Eur Urol 54: 303-314, 2008.

15. Goodison S, Rosser CJ and Urquidi V: Bladder cancer detection and monitoring: assessment of urine- and blood-based marker tests. Mol Diagn Ther 17, 71-84, 2013.

16. van Rhijn BW, van der Poel HG and van der Kwast TH: Urine markers for bladder cancer surveillance: a systematic review. Eur Urol 47: 736-748, 2005.

17. Jeong S, Park Y, Cho Y, Kim YR and Kim HS: Diagnostic values of urine CYFRA21-1, NMP22, UBC, and FDP for the detection of bladder cancer. Clin Chim Acta 414: 93-100, 2012.

18. Morgan R, Bryan RT, Javed S, et al: Expression of Engrailed-2 (EN2) protein in bladder cancer and its potential utility as a urinary diagnostic biomarker. Eur J Cancer: Feb 21, 2013 (Epub ahead of print).

19. Lei T, Zhao X, Jin S, Meng Q, Zhou H and Zhang M: Discovery of potential bladder cancer biomarkers by comparative urine proteomics and analysis. Clin Genitourin Cancer 11: 56-62, 2013.

20. Vrooman OP and Witjes JA: Urinary markers in bladder cancer. Eur Urol 53: 909-916, 2008

21. Vargová V, Pytliak M and Mechírová V: Matrix metalloproteinases. EXS 103: 1-33, 2012.

22. Paron I, Berchtold S, Vörös J, et al: Tenascin-C enhances pancreatic cancer cell growth and motility and affects cell adhesion through activation of the integrin pathway. PLoS One 6: e21684, 2011.

23. Xue Y, Li J, Latijnhouwers MA, et al: Expression of periglandular tenascin-C and basement membrane laminin in normal prostate, benign prostatic hyperplasia and prostate carcinoma. $\mathrm{Br}$ J Urol 81: 844-851, 1998.

24. Katenkamp K, Berndt A,Hindermann W, et al: mRNA expression and protein distribution of the unspliced tenascin- $\mathrm{C}$ isoform in prostatic adenocarcinoma. J Pathol 203: 771-779, 2004.

25. Parekh K, Ramachandran S, Cooper J, Bigner D, Patterson $\mathrm{A}$, and Mohanakumar T: Tenascin-C, over expressed in lung cancer down regulates effector functions of tumor infiltrating lymphocytes. Lung Cancer 47: 17-29, 2005.

26. Pauli C, Stieber P, Schmitt UM, Andratschke M, Hoffmann K, and Wollenberg B: The significance of Tenascin-C serum level as tumor marker in squamous cell carcinoma of the head and neck. Anticancer Res 22: 3093-3097, 2002.

27. Booth C, Harnden P, Selby PJ and Southgate J: Towards defining roles and relationships for tenascin- $\mathrm{C}$ and TGFbeta-1 in the normal and neoplastic urinary bladder. J Pathol 198: 359-368, 2002.

28. Berndt A, Anger K, Richter P, et al: Differential expression of tenascin-C splicing domains in urothelial carcinomas of the urinary bladder. J Cancer Res Clin Oncol 132: 537-546, 2006.

29. Richter P, Tost M, Franz M, et al: B and C domain containing tenascin-C: urinary markers for invasiveness of urothelial carcinoma of the urinary bladder? J Cancer Res Clin Oncol 135: 1351-1358, 2009. 